\title{
Demografía de la población de Anolis aquaticus (Sauria Polychrotidae) de la quebrada La Palma, Puriscal, Costa Rica
}

\author{
Cruz Marquez B. ${ }^{1}$, Lady Márquez R. ${ }^{2}$, Solanda Rea P. ${ }^{3}$ y Jefferson Márquez R. ${ }^{4}$ \\ 'Departamento de Conservación y Desarrollo Sustentable del Parque Nacional Galápagos. \\ cmarquez@spng.org.ec
}

\author{
${ }^{2}$ Escuela de Ciencias Biológicas, Pontificia Universidad Católica del Ecuador. \\ marquezrea@yahoo.es \\ ${ }^{3}$ Departamento de Ciencias, Fundación Charles Darwin, Galápagos. \\ solanda.rea@fcdarwin.org.ec \\ ${ }^{4}$ Facultad de Hotelería, Turismo, Preservación Ambiental y Gastronomía. \\ Universidad Tecnológica Equinoccial. \\ conejoloco22@hotmail.com
}

\begin{abstract}
RESUMEN: Se estudió la demografía de Anolis aquaticus (Taylor 1956), de la quebrada La Palma de Puriscal, Costa Rica $\left(9^{\circ} 45^{\prime} \mathrm{N}, 84^{\circ} 27^{\prime} \mathrm{O}\right)$. Métodos de muestreos sistemáticos mensuales en captura, marcación, recaptura, registros de depredacion y reclutamiento fueron desarrollados para la estimación poblacional. Se muestra distribución en el lugar de estudio y en todo Costa Rica. La abundancia de machos, hembras y juveniles, es estacional, es mayor en la estación seca. La proporcion de sexo en la poblacion es 1:1 y se reproducen todo el año. El tamaño poblacional fluctuo entre 86 a 575 individuos por mes. En el área de $2800 \mathrm{~m}^{2}$, se estimó una densidad entre 307 a 2053 anoles, equivalente a 11-73 individuos en cada $100 \mathrm{~m}^{2}$; y en el área de $5684 \mathrm{~m}^{2}$, se estimó una densidad entre 149 a 1008 individuos; correspondiente a 3-18 anoles en cada $100 \mathrm{~m}^{2}$, durante los meses del año. La estructura de la población es similar en machos y hembras y presenta mayor número de individuos en las categorías de tamaño grande y baja cantidad en los tamaños juveniles. Se observaron a tres especies depredando a los A. aquaticus, una de cangrejo y dos de serpientes. El reclutamiento anual es del $25-37 \%$. Los machos son reclutados en números más altos y sobreviven más que las hembras.
\end{abstract}

PALABRAS CLAVE: Demografía, Anolis aquaticus, Costa Rica 
ABSTRACTS: The demography of Anolis aquaticus (Taylor 1956) was studied at La Palma Canyon of Puriscal $\left(9^{\circ} 45^{\prime} \mathrm{N}, 84^{\circ} 27^{\prime} \mathrm{W}\right)$. A methodology based on marking, capture, recapture, predation, and recruitment was used in order to determine the population. A distribution of anoles is shown throughout the study area and the country of Costa Rica. The abundance of males, females, and juveniles is greater in the dry season. The sex ratio in the population is 1:1 and they mate throughout the year. Population abundance varies between 86-575 individuals per month. In a $2800 \mathrm{~m}^{2}$ area, a density of 307 to 2053 anoles was estimated, which is equivalent to $11-73$ individuals for each $100 \mathrm{~m} 2$. In a $5684 \mathrm{~m}^{2}$ area, a density was estimated of 149 to 1008 individuals, corresponding to 3-18 anoles for each $100 \mathrm{~m}^{2}$ during the months of the year. The population structure is similar in males and females, and it presents a greater number of individuals in adults and a low quantity of juveniles. Three species were observed as predating A. aquaticus: one crab species and two snakes. The annual recruitment rate was $25-37 \%$. Males are recruited in larger amounts and they have higher survival probabilities than females.

KEY WORDS: Demography, Anolis aquaticus, Costa Rica

\section{INTRODUCCION}

Los géneros Norops y Anolis (Sauria: Polychrotidae), comúnmente conocidos como anole se distribuyen desde Estados Unidos hasta Brasil, desde el nivel del mar hasta los 2000 msnm (Williams, 1976; Duellman, 1978; Guyer y Savage, 1986; Frost y Etheridge 1989; Savage y Guyer, 1989). Anolis está entre las lagartijas más pequeñas y numerosas del infraorden Iguania, ocupan una amplia variedad de microhabitats en todo el neotrópico, desde el arbóreo al terrestre, y aún el semiacuático. Pocas especies han sido estudiadas con detalle (Campbell, 1973; Hertz, 1975; y Rand y Rand, 1976).

Se ha estudiado el ciclo reproductivo, sobrevivencia, variación, estructura, densidades, simpatría e interrelación de los Anolis y/o Norops, tanto de N. polylepis (Peters 1873), como de $N$. limifrons (Cope, 1862) en Costa Rica y Panamá
(Andrews, 1971, 1988 y 1991, Andrews y Rand 1990), Fitch (1970, 1972, 1973 y 1975). Mientras que Fleishman (1986 y 1988) realizó estudios de comportamiento social de A. auratus en Panamá y Guyer (1986, 1988) estudió la alimentación y patrones de reproducción de $N$. humilis (Peters, 1863) en Costa Rica. En las 21 especies de Norops y cinco de Anolis de Costa Rica analizadas por Taylor (1956), el parche poblacional de la quebrada $\mathrm{La}$ Palma, corresponde a la especie de Anolis aquaticus (Taylor 1956).

La lagartija $A$. aquaticus de Costa Rica, presenta amplia distribución en la vertiente del Pacifico hacia el Sur y centro del país (La Palma, Puriscal, San Jose) hasta el Sur cerca de los límites con Panamá (San Vito y Golfito). Esta especie no ha sido registrada al norte del país, probablemente por la aridez de la región (Márquez 1994, Márquez et al 2005). Es una lagartija semiacuática y prefiere los ambientes 
muy húmedos por lo cual habita en los arroyos, para evadir la depredación. Esta especie, aun no ha sido registrada en otros paises de Sur, Centro y Norte America. Su población es más abundante en la época seca; misma que disminuye en la estación lluviosa de Costa Rica. Mantiene una proporción sexual anual 1:1. El tamaño poblacional de la especie es variable durante los meses del año; como también varia la densidad (Márquez 1994).

Estudios previos registran depredación de $A$. aquaticus por serpientes, cangrejos y aves como el pájaro bobo, mismos que limitan el reclutamiento de jóvenes, y por consiguiente el reclutamiento es disminuido al 25-37\% anual (Márquez 1994). No existen estudios detallados acerca de la demografía de la especie en Costa Rica, por lo que este estudio persigue proporcionar información más detallada acerca de la distribución de la especie, tamaño y estructura de la población, involucrando otros aspectos como información sobre la extensión potencial del hábitat, incluyendo también algunos parámetros reproductivos, nacimiento, depredación y mortalidad natural de jóvenes y adultos machos y hembras.

\section{MATERIALES Y METODOS}

\section{Área de Estudio}

La quebrada La Palma de Puriscal, nace en las estribaciones de los montes de Turrubares en el sector Norte de la cordillera de Talamanca y desemboca en el río Quibel. En la estación seca su cauce mide $0,89 \pm 0,30 \mathrm{~m}$ en promedio (de $0,30-1,44$, $\mathrm{n}=15$ mediciones); en la época lluviosa, su cauce mide $1,09 \pm 0,34 \mathrm{~m}$ en promedio ( $0,50-1,64, \mathrm{n}=15$ mediciones). A lo largo de la quebrada existe un bosque ripario, arbóreo-arbustivo y herbáceo que comienza desde el borde del agua hasta 50-100 m fuera de la quebrada, seguido por potreros en ambos márgenes.

El sitio de estudio se encuentra localizado, según el sistema de zonas de vida de Holdrige, en el bosque húmedo Premontano (Valerio, 1991). Según los tipos de vegetación de Costa Rica (Gómez, 1986), se clasifica entre las formaciones estacionales con un bosque tropical húmedo de altura media. En cuanto a clima se presenta entre la región de clima húmedo con estación lluviosa moderada y una estación seca corta (Herrera, 1985).

\section{Métodos Usados en el Campo}

La investigación se llevó a cabo de marzo 1991 a marzo 1993, en la quebrada La Palma de Puriscal, ubicada a $16 \mathrm{~km}$ de Santiago de Puriscal por la vía a Puerto Quepos, Provincia de San José, Costa Rica $\left(9^{\circ} 45^{\prime} \mathrm{N}, 84^{\circ} 27^{\prime} \mathrm{O}\right)$, a una altitud de 910 msnm (Fig.1). Se trabajó con 297 individuos incluidos machos, hembras y juveniles naturales. 


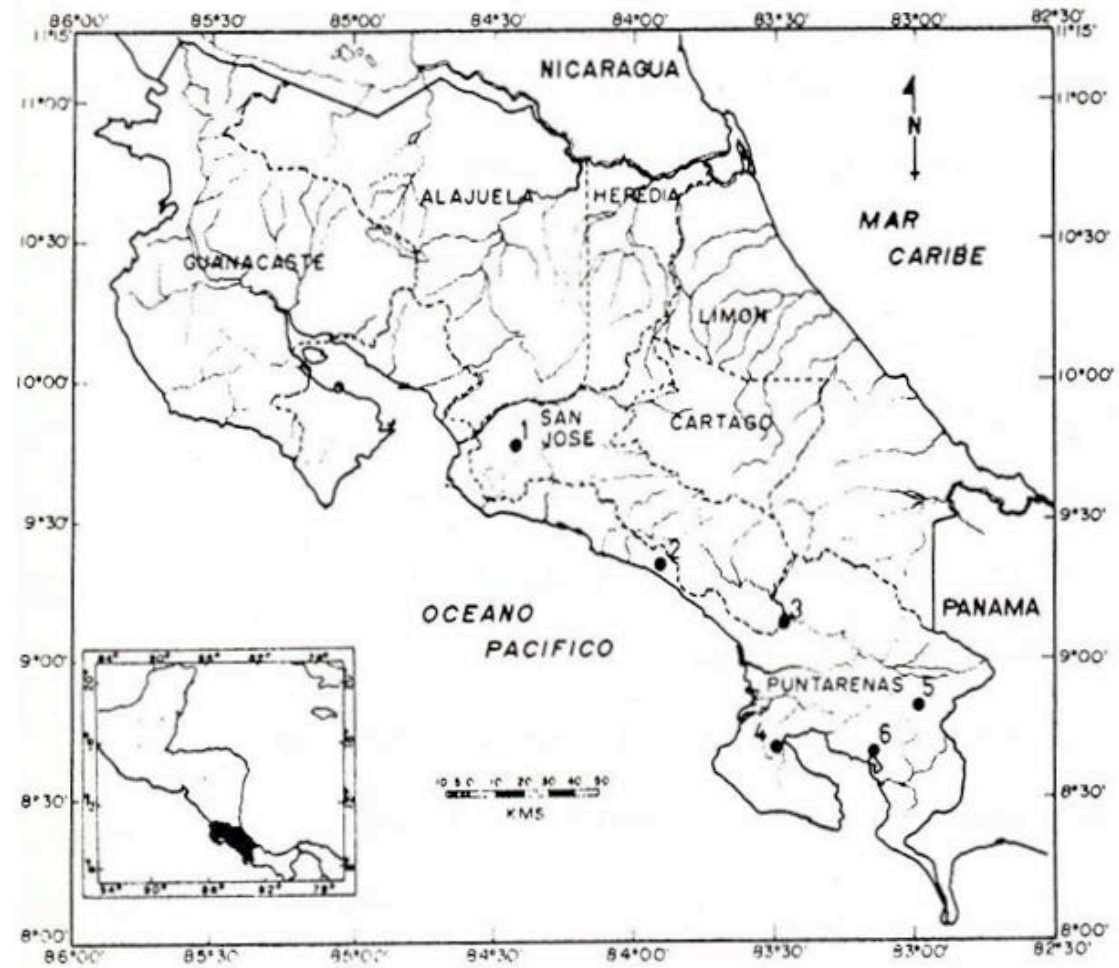

Figura 1. Ubicación del sitio de estudio y distribución de Anolis aquaticus en Costa Rica (1) quebrada La Palma de Puriscal, (2) Playa Dominical, (3) Palmar Norte, (4) Rincón de Osa, (5) Las Cruces, San Vito, (6) Golfito.

Para estimar la población y densidad se consideró dos tipos de área: 1) Una de $406 \mathrm{~m}$ de largo por $14 \mathrm{~m}$ de ancho, siete metros por lado $\left(406 \mathrm{~m} \times 14 \mathrm{~m}=5684 \mathrm{~m}^{2}\right)$, que incluyó a toda el área revisada de la quebrada en donde las lagartijas estaban más dispersas. 2) Una longitud de $200 \mathrm{~m}$ por $14 \mathrm{~m}$ de ancho $(200 \mathrm{~m} \times 14 \mathrm{~m}=2800$ $\mathrm{m}^{2}$ ) en donde los animales estuvieron más agrupados durante el año. También en las capturas y recapturas fue medida la distancia al agua a que se encontró cada individuo capturado.

Se utilizó el método de marca y recaptura de Jolly (Krebs 1999). Para marcar los animales como referencia se utilizaron los métodos de marcación de Tinkle (1967) y Medica et al. (1971). Para marcar más animales y cortar menos uñas de los dedos de los individuos, se modificaron estos dos métodos de la siguiente manera: se asignó a los dedos de la pata anterior derecha los números 2, 4, 6 y 8; a los dedos de la pata anterior izquierda los números $1,3,5,7$; a los dedos de la pata posterior derecha los números 20, 40,60, 100; y la pata posterior izquierda los números $30,50,70,200$. Con una tijera se cortó la uña del dedo correspondiente de cada pata de los animales. 
Los Anolis fueron medidos, marcados y liberados durante dos días de cada mes por el lapso de 18 meses a lo largo de los dos ramales de la quebrada (Fig. 2). Después de 25 ó 30 días, se realizó la captura, marca y recaptura de los individuos marcados y no marcados. Transectas transversales de 15 m cada una, fueron realizadas a los lados de la quebrada, para medir distancia de alejamiento de los anoles desde el agua hacia afuera en la quebrada. Para observar donde perchaban las lagartijas para dormir, fueron buscadas en la noche en los mismos lugares donde se los buscaba durante el día, $\mathrm{y}$ fueron vistos dormir en pequeñas y grandes cascadas que se encontraban en el arroyo. Depredadores presentes en el área de estudio fueron observados $\mathrm{y}$ registrados minuciosamente.

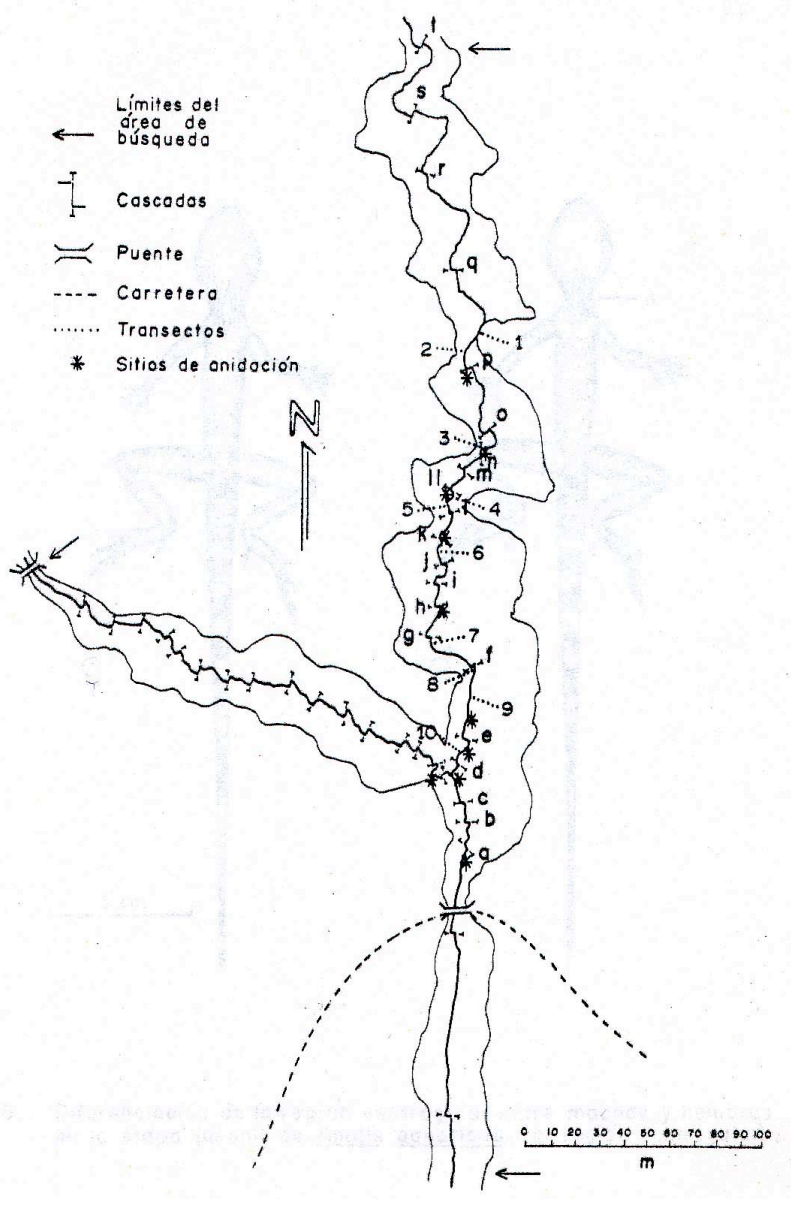

Figura 2. Área de estudio, quebrada La Palma, Puriscal (a-f= distribución de las cascadas) 
Para registrar las condiciones ambientales en el lugar de estudio, se instaló un pluviómetro y un termómetro de máxima y mínima; en los días de muestreo se usó un sicrómetro para medir la humedad relativa. La estación meteorológica más cercana se encuentra en Santiago de Puriscal a $16 \mathrm{~km}$ de la quebrada. La estación seca corresponde a los meses de diciembre-abril $(<300 \mathrm{~mm}$ de precipitación) y la lluviosa de mayo-noviembre ( $>300 \mathrm{~mm}$ de precipitación). Los dos años de estudio tuvieron una precipitación promedio mensual diferente, con picos en mayo-septiembre de 1991 (promedio anual $=2103 \mathrm{~mm}$ ) y junio-octubre de 1992 (promedio anual = $2279 \mathrm{~mm}$ ) (Fig. 3). Los promedios mensuales de la humedad relativa dentro y fuera de la quebrada en los meses de estudio fueron variables (Fig. 4). Asimismo fueron los promedios mensuales de las temperaturas máxima, mínima y media en el sitio de estudio en 1992 y enero-marzo 1993 (Fig. 5).

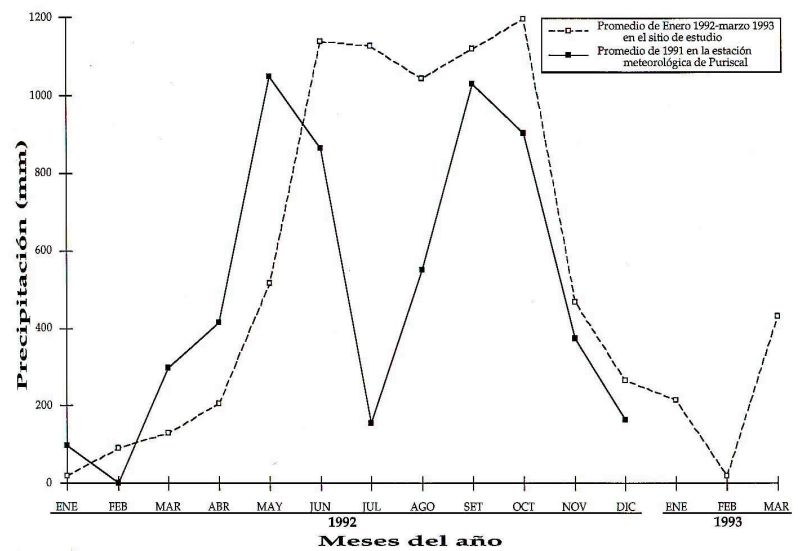

Figura 3. Precipitación mensual en 1991 en la estacion meteorologica de Puriscal, de enero a diciembre de 1992 y de enero a marzo de 1993, en la quebrada La Palma de Puriscal.

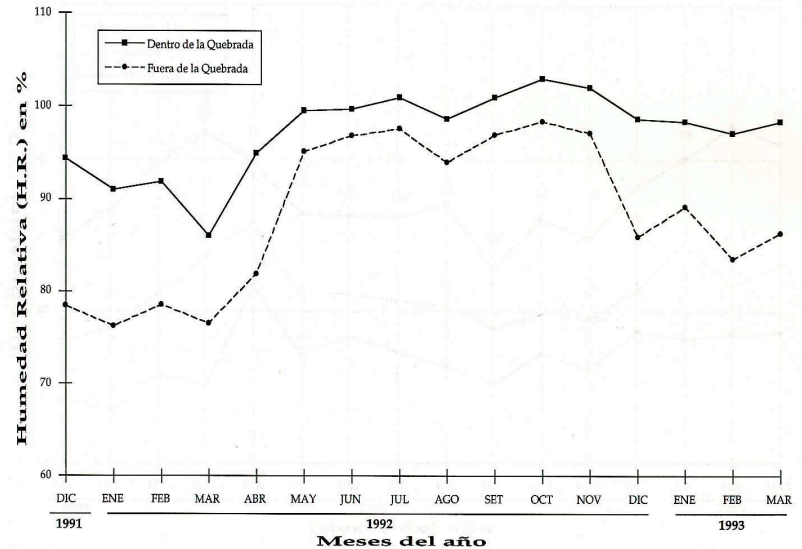

Figura 4. Humedad Relativa (H.R.) promedio mensual de diciembre de 1991, enero a Diciembre 1992 y enero marzo de 1993, en la quebrada La Palma de Puriscal. 


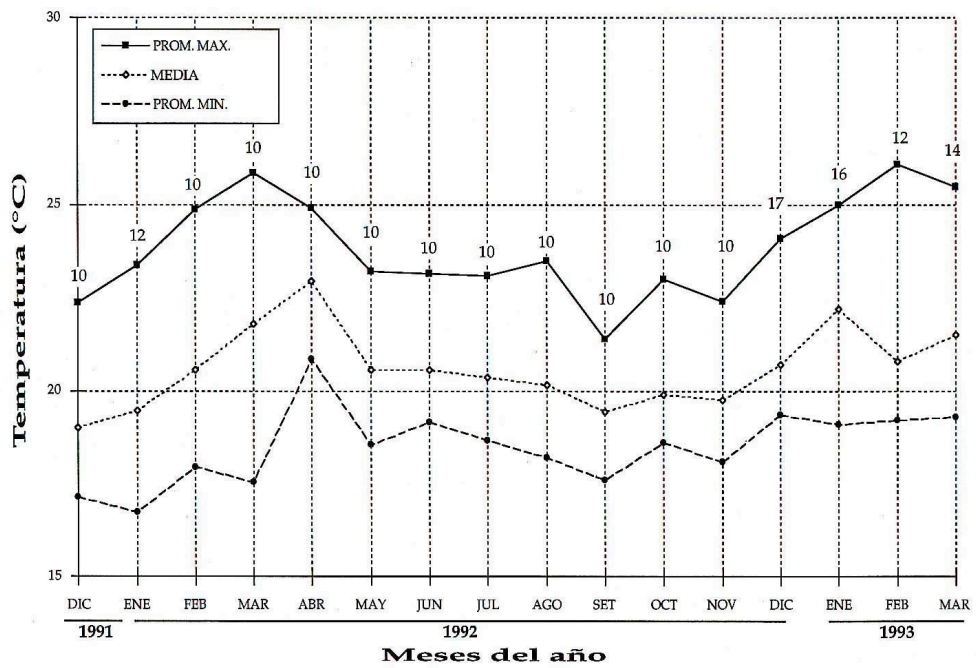

Figura 5. Temperatura promedio maximas, minimas y ambiental, de diciembre de 1991, Enero a diciembre de 1992 y enero a marzo de 1993, en la quebrada La Palma de Puriscal.

\section{Análisis estadístico}

Se utilizó el método de captura, marca y recaptura de Jolly (Krebs 1999) para la estimación de la población. El análisis estadístico, se llevó a cabo por medio del paquete SPSS y con la prueba de Ji-cuadrado, se hizo la fluctuación de la población, proporción sexual y estructura poblacional. Correlación, para la relación entre la abundancia y la precipitación y Tukey, para comparar la extensión potencial del hábitat entre la estación seca y lluviosa de los machos, las hembras y los juveniles (Zar 1999).

\section{RESULTADOS}

\section{Distribución}

Una revisión de las colecciones del Museo de Zoología de la Universidad de Costa Rica, comunicaciones personales de F. Valverde y F. Bolaños (1993), así como las colecciones de Taylor (1956), identificaciones y ubicación geografica en la herpetofauna de Costa Rica por Savage y Villa (1986), permitieron determinar la distribución de A. aquaticus en Costa Rica (Fig. 1). Las graficaciones de los animales capturados y recapturados en un mapa de la quebrada, hizo posible mostrar la distribución de la especie en el lugar de estudio. Estas lagartijas, en promedio, no se alejaron más alla de 1,6 $\mathrm{m}$ del agua en la época seca y 2,0 $\mathrm{m}$ en la época lluviosa (Tabla 1 y Fig. $6)$. Se compararon las muestras entre machos, hembras y juveniles, y no fueron diferentes (Tukey $=\mathrm{P}>0,05$ ). 


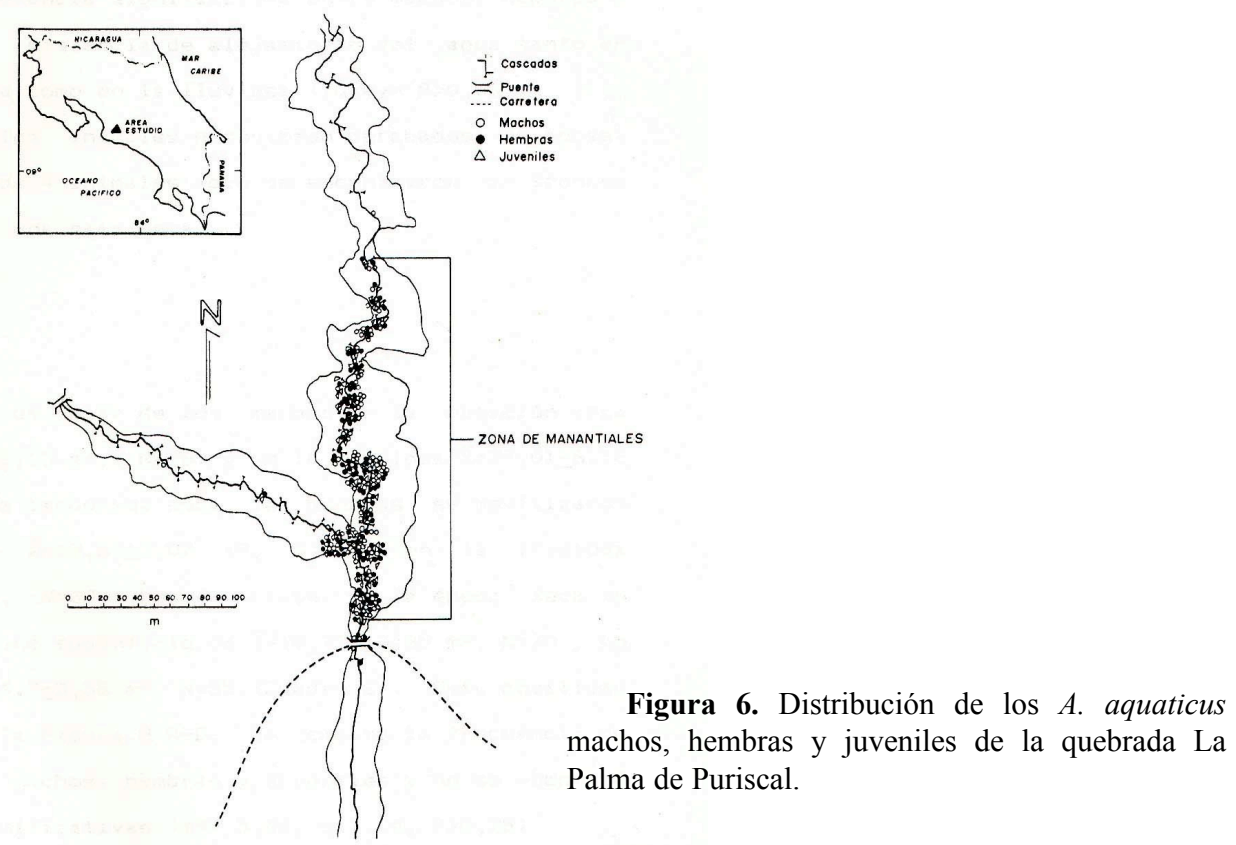

Tabla 1. Promedios de la distancia a que se encontró A. aquaticus desde el agua hacia fuera (extensión potencial del hábitat), en la época seca (diciembre a abril) y lluviosa (mayo a noviembre), en la quebrada La Palma de Puriscal ( $\mathrm{n}=$ número de individuos por categoría).

\begin{tabular}{|c|c|c|c|c|}
\hline Estaciones & Sexo & n & $\overline{\mathbf{X}} \pm \mathbf{D E}(\mathbf{m})$ & Rangos (m) \\
\hline Seca & $\sigma^{\prime}$ & 43 & $1,64 \pm 0,45$ & $0,04-4,90$ \\
\hline$"$ & q & 45 & $1,44 \pm 0,51$ & $0,10-3,90$ \\
\hline$"$ & $\mathrm{~J}$ & 70 & $1,20 \pm 0,56$ & $0,04-3,80$ \\
\hline Lluviosa & $\sigma^{\prime}$ & 40 & $2,04 \pm 0,38$ & $0,25-7,0$ \\
\hline$"$ & q & 39 & $1,61 \pm 0,63$ & $0,05-5,20$ \\
\hline$"$ & $\mathrm{~J}$ & 55 & $1,51 \pm 0,45$ & $0,05-3,90$ \\
\hline
\end{tabular}

\section{Estado Poblacional}

Los animales fueron más abundantes en la época seca tanto machos como hembras y juveniles; la cantidad de lagartijas capturadas entre noviembre a diciembre y de enero a febrero, fueron menores. Las muestras siempre fueron más pequeñas en la época lluviosa, a excepción de la muestra de julio de 1991; que fue la más alta de todo el muestreo (Tabla 2). 
Tabla 2. Estimaciones de densidad y tamaños mensuales de población de A. aquaticus de la quebrada La Palma de Puriscal, San José, Costa Rica.

\begin{tabular}{lllll}
\hline Mes/año & n. observada & N. Estimada & $\begin{array}{l}\text { Densidad } \\
\text { Anolis/5684m }\end{array}$ & $\begin{array}{l}\text { Densidad* } \\
\text { Anolis/2800m }\end{array}$ \\
\hline Marzo 1991 & 26 & 0 & 0 & 0 \\
Julio & 58 & 186,7 & 334 & 667 \\
Diciembre & 41 & $161,3^{* *}$ & $282^{* *}$ & $576^{* *}$ \\
Enero 1992 & 61 & $574,8^{* *}$ & $1008^{* *}$ & $2053^{* *}$ \\
Febrero & 51 & $306^{* *}$ & $537^{* *}$ & $1093^{* *}$ \\
Marzo & 43 & 379,9 & 667 & 1357 \\
Abril & 37 & 273,2 & 478 & 975 \\
Mayo & 17 & 104,6 & 184 & 375 \\
Junio & 19 & 256 & 449 & 914 \\
Julio & 23 & 336 & 589 & 1200 \\
Agosto & 20 & 102 & 179 & 364 \\
Septiembre & 16 & $157,3^{* *}$ & $275^{* *}$ & $561^{* *}$ \\
Octubre & 16 & $122,5^{* *}$ & $216^{* *}$ & $439^{* *}$ \\
Noviembre & 21 & $226,3^{* *}$ & $396^{* *}$ & $807^{* *}$ \\
Diciembre & 31 & $362,7^{* *}$ & $635^{* *}$ & $1293^{* *}$ \\
Enero 1993 & 29 & $174^{* *}$ & $305^{* *}$ & $621^{* *}$ \\
Febrero & 34 & $85,6^{* *}$ & $149^{* *}$ & $307^{* *}$ \\
Marzo & 23 & 0 & 0 & 0 \\
\hline
\end{tabular}

$\mathrm{X} 2=453,0$, g. $1 .=15, \mathrm{P}<<0,0001$.

$*$ = Mayor densidad de la población, $\mathrm{n}=$ Muestras, $\mathrm{N}$ = Población

** = Estimaciones y densidades en la época seca

Proporción de sexo de la Muestra

En la muestra total capturada incluia 83 machos (43 en la epoca seca y 40 en la 1luviosa) y hembras 84 (45 en la época seca y 39 en la lluviosa) y entre los meses, mostraron que no existía equilibrio $1: 1$ en la proporción sexual, pero en la muestra total en machos y hembras fueron iguales $\mathrm{y}$ mostraron relación $1: 1$, no hubo diferencia entre las muestras en las dos épocas del año $\mathrm{y}$ entre los sexos $\left(\mathrm{X}^{2}=3,74\right.$, g. $1 .=1, \mathrm{P}>$ $0,05)$.

\section{Densidad y Fluctuación del Tamaño Poblacional}

Durante el año, los tamaños poblacionales estimados fluctuaron entre 86 a 575 individuos por mes y las densidades de la 
población, fueron calculadas en dos tamaños de área: 1. Con $5684 \mathrm{~m}^{2}$ que incluía toda el área de estudio y de menor densidad, y 2. Con $2800 \mathrm{~m}^{2}$, que contenía solamente el área de mayor densidad. Los cálculos en el área de menor densidad variaron desde 149 en febrero de 1993 y 1008 en enero de 1992 (149 y 1008 individuos; correspondiente a 3-18 anoles por cada 100 $\mathrm{m}^{2}$ ). En el área de mayor densidad hubo 307 y 2053 animales por cada $2800 \mathrm{~m}^{2}$ equivalente a 11-73 lagartijas por cada 100 $\mathrm{m}^{2}$ (Tabla 2). Los tamaños y densidades poblacionales entre las áreas de mayor y menor densidad fueron diferentes $\left(\mathrm{X}^{2}=\right.$ $453,0$, g. $1 .=15, \mathrm{P}<<0,0001)$.

La estimación del tamaño mensual de la población mostró que ésta fluctúa mes a mes durante el año, que es más alta en la estación seca. Hubo diferencia significativa entre los valores estimados (población mensual estimada) frente a los valores esperados (promedio de los valores

$$
\mathrm{n}=297(151 \mathrm{M} \text { y } 146 \mathrm{H})
$$

estimados; $\mathrm{X}^{2}=453,0$ g. $1 .=15, \mathrm{P}<<$ 0,0001; (Tabla 2).

\section{Estructura poblacional}

Los A. aquaticus en la quebrada La Palma, presentaron una estructura poblacional con tres picos, uno por cada estrato en tres categorías de tamaño. En la categoría entre los 35-40 mm los juveniles, entre los 60-65 mm las hembras y 70-75 $\mathrm{mm}$ los machos (Fig 7). En ninguno de los niveles de tamaño, la relacion de sexo fue 1:1; se exceptua la categoría de 45-50 que machos y hembras estuvieron proximos a 1:1. Se encontró diferencia muy significativa en la estructura de la población $\left(\mathrm{X}^{2}=564 \mathrm{~g} .1 .=\right.$ $16, \mathrm{P}<<0,0001)$ entre machos y hembras. Los niveles de 70,5 hasta 80,4 presentaron solo machos; la longitud corporal máxima que alcanzan las hembras es de $68 \mathrm{~mm}$ y los machos hasta de $80,4 \mathrm{~mm}$.

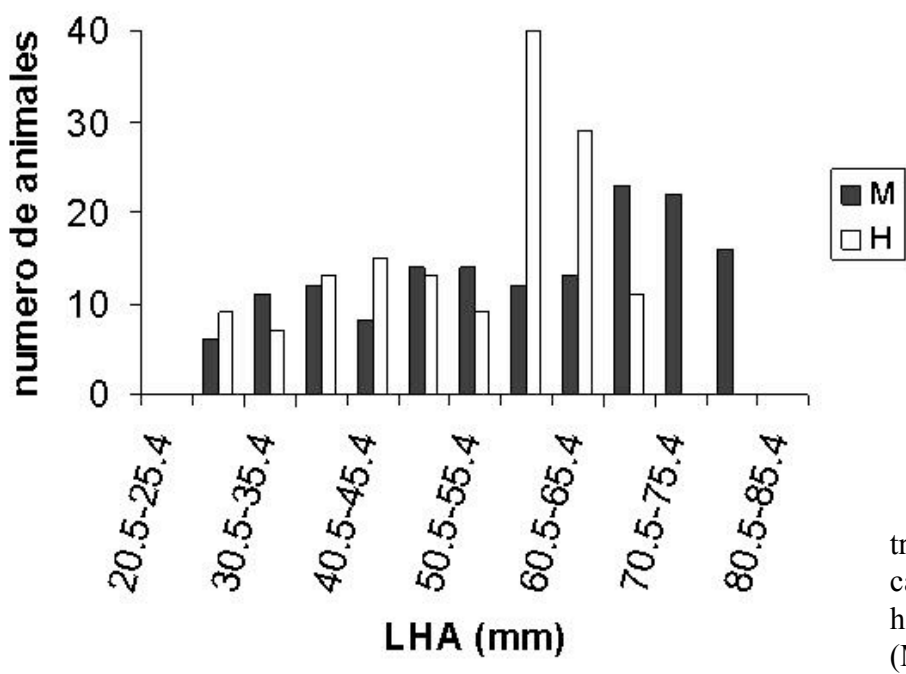

Revista Ecuatoriana de Medicina y Ciencias Biológicas - Vol. XXX Números 1 y 2: 62-77, octubre 2009
Figura 7. Estructura de la muestra poblacional de los A. aquaticus capturados y medidos entre 1991 hasta 1993 de la quebrada La Palma ( $\mathrm{M}=$ machos, $\mathrm{H}=$ hembras). 
En las noches, se observó y registró a animales durmiendo en los espacios bajo las pequeñas cascadas. Tanto los capturados durante el día, como los que se vieron durmiendo en la noche bajo las cascadas, fueron más abundantes en la estación seca y menos en la lluviosa. Existe una relación negativa significativa $(\mathrm{r}=0,77$, g. $1 .=12, \mathrm{P}$ $<0,002)$ entre la precipitación y la abundancia nocturna y diurna de los anoles en la quebrada (Fig. 8).

\section{Depredadores}

Se observó a dos serpientes comer $A$. aquaticus, Chironius grandisquamis, se comió a un individuo en febrero de 1992 y Leptodeira septentrionalis lo hizo en diciembre de 1992. Un cangrejo de río (Ptychophalus tristanis) se comió a una lagartija hembra en abril de 1992. Otras especies de depredadores, que fueron registrados por otros autores estudiando otras lagartijas del género Norops y Anolis, no fueron observadas comer a esta epecie de lagartija en la quebrada (Tabla 3).

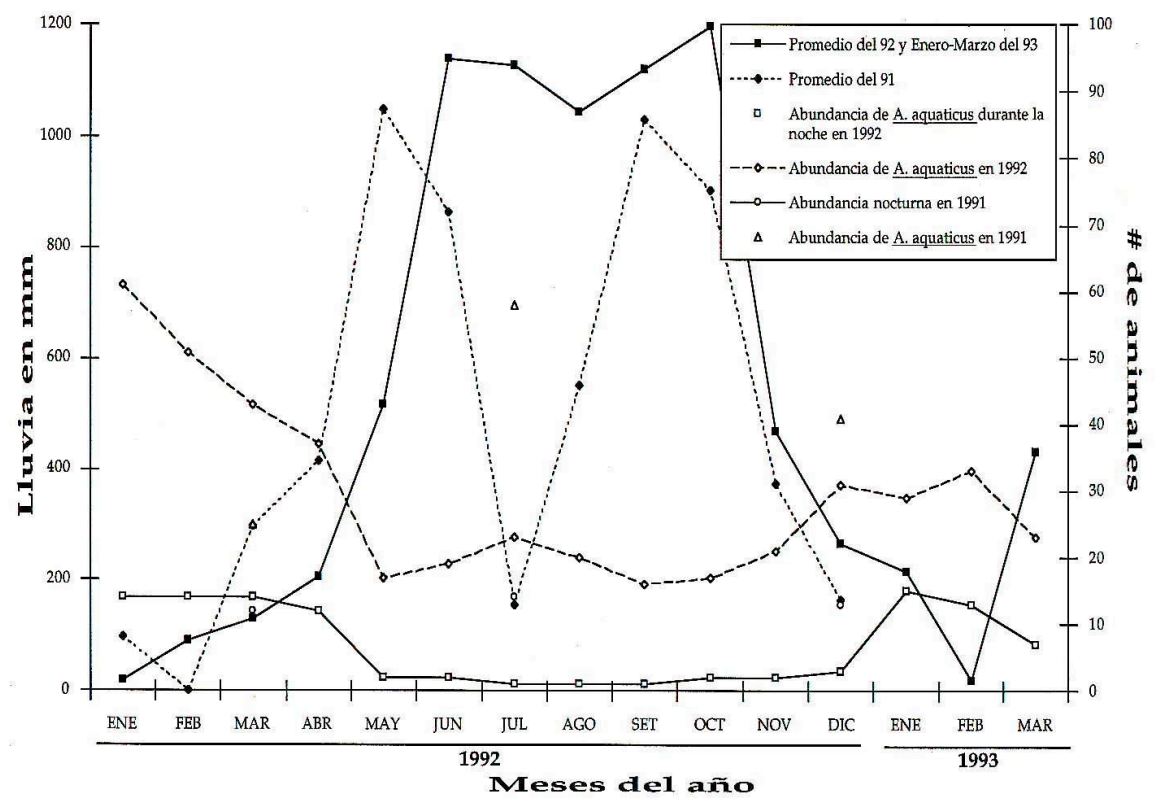

Figura 8. Abundancia de A. aquaticus en relacion con la precipitación promedio mensual en la estacion meteorologica de Puriscal, de 1991, enero a diciembre de 1992 y enero a marzo de 1993 en la quebrada La Palma de Puriscal. 
Tabla 3. Depredadores potenciales de A. aquaticus observados en la quebrada La Palma de Puriscal.

\begin{tabular}{|c|c|c|}
\hline Nombre Común & Familia & Nombre Científico \\
\hline Serpiente & Colubridae & Oxybelis aeneus \\
\hline ، & “ & *Chironius grandisquamis \\
\hline “ & “ & Geophis \\
\hline “ & “ & *Leptodeira septentrionales \\
\hline “ & “ & Ninia sebae \\
\hline “ & “ & Spilotes pullatus \\
\hline “ & “ & Drymobius margaritiferus \\
\hline Gallego & Basiliscinae & Basiliscus plumifrons \\
\hline Cangrejo & Pseudothel Pusidae & *Ptychophalus tristanis \\
\hline Bobo & Momotidae & Momotus momota \\
\hline
\end{tabular}

*= Especies que se observaron depredar A. aquaticus en la quebrada La Palma.

Reclutamiento (Adición de jóvenes en la población) y permanencia de juveniles, machos y hembras en el área de estudio

El reclutamiento de machos juveniles fue $37 \%$, y el de las hembras $25,4 \%$, durante los 18 meses de muestreo. La permanencia registrada en el área de estudio para juveniles y machos adultos fue de 51,6\%, de hembras jóvenes y adultas de $48,4 \%$. La permanencia mínima registrada en el área de estudio, para machos y hembras fue de un mes (dos recapturas). La recaptura máxima fue de ocho veces en 17 meses, para los machos y de siete veces en 17 meses para las hembras.

\section{DISCUSION}

\section{Distribución}

Las lagartijas riparias A. poecilopus y A. lionotus estudiadas por Campbell (1973) en Panamá y A. megalopithecus estudiada por Rueda (1989) en Colombia, no se ale- jan más de $3 \mathrm{~m}$ del agua. Un comportamiento similar se observó en $A$. aquaticus en la quebrada La Palma de Puriscal, en donde la máxima distancia promedio del animal al agua fue $2 \mathrm{~m}$ en la época lluviosa y solo 1,6 $\mathrm{m}$ en la época seca. Esto indica, que el ámbito de hogar en la época seca, fue más pequeño que en la época lluviosa para los machos, las hembras y los juveniles. En lo referente a que durmieron bajo los espacios vacíos, que se formaban entre el substrato y las grandes cascadas de agua en la quebrada, para protegerse de los depredadores nocturnos y evitar ser presa fácil de los mismos. En lo referente a la distribución de la especie en el país, se registra en las localidades; de la quebrada La Palma de Puriscal, Playa Dominical, Palmar Norte, Rincón de Osa, Las Cruces y Golfito; son regiones de mayor humedad en el ambiente en el país, y la región Noroeste es más seca, particularmente Guanacaste (Comn. Pers. F. Bolaños, F. Valverde 1993, Savage y Villa 1986 y Taylor 1956). 


\section{Estado Poblacional}

La abundancia de los A. aquaticus, es baja en la epoca lluviosa por la mayor dispersión de los individuos a más distancia del agua en la quebrada y en la temporada seca se encuentran más agrupados y más cercanos al agua; tanto con relación a los margenes de la quebrada como con relación a lo largo de la quebrada. Son reptiles que deben vivir alrededor de mucha humedad en el ambiente y siempre deben permanecer cerca del agua, para evadir al depredador entrando rápidamente al agua (Márquez 1994, Márquez et al 2005, Savage y Guyer 1989).

\section{Proporción sexual Poblacional}

La muestra capturada mostro que existía un equilibrio 1:1 en la proporción sexual de la población entre meses y estaciones del año. Esto sugiere que: 1) Tanto los machos como las hembras, podrían estar sufriendo igual porcentaje de depredación anual. 2) Que el porcentaje de nacimientos de crías son proporcionales entre los sexos en cada año. 3) En la quebrada La Palma de Puriscal la temperatura ambiental de incubación de los huevos produce una prole con igual proporción sexual. En lo referente a demografía poblacional, es un aspecto de estudios que no han sido realizado en $A$. aquaticus y existen pocos en otras especies.

\section{Densidad y Fluctuación del Tamaño Poblacional}

La población de A. aquaticus, no presenta una relación de sexo 1:1 en todas las categorías, a excepción del nivel 45-50 que es próxima a $1: 1$; sin embargo en la muestra general, es 1:1. Lo anterior sugiere que en los últimos niveles de tamaño, es donde se reúne el mayor número de hembras y machos de la población. Se asume que la mayor abundancia que presentan los machos y las hembras, en los tamaños mayores, es producto de algunos años de reclutamiento acumulado.

La cantidad de animales capturados y recapturados así como también el número de individuos que se observa durmiendo bajo las cascadas durante la noche, se correlaciona en forma negativa con la precipitación durante este estudio. Esto indica que a mayor precipitación, hay una menor cantidad de individuos que se exhiben en las perchas, lo cual posiblemente se deba, a que ellos, permanecen más tiempo dentro de las grietas, por las bajas temperaturas que se registran en la época lluviosa, ocacionando mayor dispersión en el área.

Los estimados de densidad poblacional fueron menores (149 y 1008 individuos) al incluir las áreas de mayor y menor densidad de los A. aquaticus de la quebrada La Palma. En el sector en que hubo menos penetración solar (bosque más denso con manantiales permanentes en los abruptos márgenes de la quebrada), los Anolis estaban más agrupados lo que dio como resultado densidades más altas. Esto sugiere que lagartijas usan con más frecuencia los lugares con mayor humedad y poco o nada son usados los sitios donde ocurre ausencia de humedad. Este dato afirma que estos animales son acuáticos y que no pueden vivir más alla de los dos metros fuera del agua.

En lo referente a las densidades, Campbell (1973), estudió A. poecilopus y A. lionotus (Cope 1861) en Panamá, Tri- 
vers (1976), A. garmani en Jamaica, Andrews y Rand (1990), N. limifrons (Cope 1862), en Barro Colorado, todos estos autores estimaron densidades de población de las diferentes especies anotadas, desde 1 anolis $/ 10 \mathrm{~m}^{2}$ a 1 anolis $/ 45 \mathrm{~m}^{2}$, para la temporada húmeda y 1 anolis $/ 40 \mathrm{~m}^{2}$ hasta 1 anolis $/ 75 \mathrm{~m}^{2}$ en la época seca respectivamente. Sin embargo, en este estudio, se estimó, desde 3-18 anoles en cada $100 \mathrm{~m}^{2}$, donde lagartijas eran menos densas, y 11-73 individuos en cada $100 \mathrm{~m}^{2}$, donde $A$. aquaticus era más denso; son densidades ligeramente mayores a las encontradas por los autores arriba indicados. Campbell (1973) comunica que las densidades en lagartijas riparias varían de acuerdo a la cantidad de luz que penetra al piso de la quebrada. A. aquaticus de este estudio; no solamente requiere de penetración solar, sino tambien de abundante humedad en el ambiente circundante.

\section{Estructura Poblacional}

El mayor pico de abundancia lo muestran las hembras adultas en las categorías entre los 55 a los $65 \mathrm{~mm}$ de LHA, y mediana cantidad presenta el nivel arriba de los $65 \mathrm{~mm}$ de longitud corporal. Bajos números de individuos muestran las hembras juveniles, por debajo de los $50 \mathrm{~mm}$ de tamaño. Tambien los machos adultos, indican alta abundancia en los $65 \mathrm{~mm}$ de LHA, pero bajos números presentan los adultos, subadultos y jóvenes en las categorías entre los 30 hasta los $65 \mathrm{~mm}$ de LHA. Esto sugiere que no muchos individuos machos y hembras alcanzan la madurez sexual. Un número no determinado de anoles, no se reclutan, debido a depredacion o por muer- te natural accidental. Andrews 1988, sostiene que las camadas de animales jóvenes $\mathrm{y}$ adultos sufren disminución poblacional por depredacion y mortalidad natural.

\section{Depredación}

En la quebrada La Palma, los depredadores de $A$. aquaticus fueron dos especies de serpientes (Chironius grandisquamis y Leptodeira septentrionalis) y una especie de cangrejo (Ptychophalus tristanis). Durante este estudio, no se observó comer a $A$. aquaticus a ninguno de los depredadores mencionados por otros autores indicados más adelante.

Campbell (1973) anota que los depredadores potenciales para las especies semiacuáticas, $A$. poecilopus y $A$. lionotus fueron Oxybelis brevirostris, peces no identificados, Nasua narica y Chironius carinatus; asi mismo menciona a un $A$. poecilopus adulto que comió a un recién nacido de su misma especie. Andrews, (1971) y (1979), observó que $N$. polylepis, $N$. limifrons y otros anoles eran depredados por mantis, el ave Momotus momota, algunas especies de serpiente y Basiliscus plumifrons. Campbell (1973), Trivers (1976), Andrew y Rand (1990), mencionan a otros depredadores de especies de Norops y Anolis. Según Savage y Guyer 1989, los A. aquaticus, son especies que se encuentran cerca de corrientes de agua y se adentran en ella en huída del depredador. Leal y Lossos 2000, con relación a este mismo comportamiento sugieren que $A$. aquaticus con relación a los otros Anolis, ha evolucionado independientemente dos veces en el Caribe y dos veces en el continente Centro Americano. 


\section{Reclutamiento}

Los juveniles de $A$. aquaticus quedan libres después de eclosionar y salen al exterior de las grietas (nidos), el mismo día que nacen; ya que, muchos de los huevos no son enterrados, sino que son dejados en fisuras de troncos de madera en descomposición o suspendidos en fisuras y grietas en las abruptas paredes de la quebrada. Una vez en el exterior, los juveniles se enfrentan a depredadores entre otros pueden ser vertebrados, incluso los adultos de su misma especie e invertebrados como la Mantis religiosa y cangrejos. Luego de atravesar un sinnúmero de escollos depredativos, son pocos los que alcanzan la edad adulta (Andrews 1971, 1979; Andrews y Rand 1990; Márquez et al 2005).

\section{CONCLUSIONES}

- Se estimó, desde 2-10 anoles en cada $100 \mathrm{~m} 2$, donde la población de lagartijas era menos densa, y 11-73 individuos en cada $100 \mathrm{~m} 2$; donde A. aquaticus era más denso.

- La estructura poblacional es similar en machos y hembras y presentan mayor número de individuos en las categorías adultas

- La abundancia de machos hembras y juveniles de $A$. aquaticus es estacional y es mayor en la estación seca.

\section{AGRADECIMIENTOS}

Nuestra sincera gratitud a las familias Robinson y Porras por permitirnos vivir y realizar todo el estudio en sus propiedades. Al Dr. William Eberhard por sus valiosos comentarios al manuscrito. A todas y cada una de las personas que nos ayudaron de una $u$ otra manera a terminar esta investigación.

\section{REFERENCIAS BIBLIOGRÁFICAS}

Andrews, R. M. 1971. Structural habitat and time budget of a tropical Anolis lizard. Ecology 52:262-270.

Andrews, R. M. 1979. Reproductive effort of female Anolis limifrons (Sauria: Iguanidae). Copeia 1979:620-626.

Andrews, R. M. 1988. Demographic correlates of variable egg survival for a tropical lizard. Oecología 76:376-382.

Andrews, R. M. and Rand, A. S. 1990. Reproducción estacional y fluctuaciones poblacionales a largo plazo de la lagartija Anolis limifrons. pp. 469-476. En: Leigh E. G., Jr., A.S. Rand y D. M. Windsor (Eds). Ecología de un bosque tropical, ciclos estacionales y cambios a largo plazo. Editorial Presencia Ltda. Colombia.

Andrews, R. M. 1991. Norops polylepis (Lagartija, Anole, Anolis Lizard) pp. 409410. En: Janzen, D. H., (Ed). Historia Natural de Costa Rica. Editorial Universidad de Costa Rica, Costa Rica.

Campbell, H. W. 1973. Ecological observations on Anolis lionotus and Anolis poecilopus (Reptilia, Sauria) in Panamá. Am. Mus. Nat. Hist. Univ. Florida. No. 2516 N. Y. 29 p.

Krebs. C. J. 1999. Ecological Methodology. University of British Columbia. Harper \& Row, Publishers. New York. 
Fitch, H. S. 1970. Reproductive Cycles of lizards and snakes Occasional Papers. Museum of Natural History, University of Kansas 52:1-247.

Fitch, H. S. 1973. Population structure and survivorship in some Costa Rican lizards.

Occasional Papers. Museum of Natural History, University of Kansas 18:1-41.

Fitch, H. S. 1975. Sympatry and interrelationships in Costa Rica anoles. Occasional Papers. Museum of Natural History, University of Kansas 40: 1-60.

Fleishman, L. J. 1986. Motion detection in the presence and absence of Background motion in an Anolis lizard. Jour. Comp. Physiol. 159:711-720.

Fleishman, L. J. 1986. 1988. The social behavior of Anolis auratus a grass Anole from Panamá. J. Herp. 22:13-23.

Gómez, L. D. 1986. Vegetación de Costa Rica. Editorial Universidad Estatal a Distancia. San José, Costa Rica. 327 p.

Guyer, C. 1986. Seasonal patterns of reproduction of Norops humilis (Sauria:Iguanidae) in Costa Rica. Rev. Biol. Trop. 34:247-251.

Guyer, C. 1988. Food supplementation in a tropical Mainland Anoles, Norops humilis effect on individuals. Ecology 69:362-369.

Herrera, W. 1985. Clima de Costa Rica. Editorial Universidad Estatal a Distancia. San José, Costa Rica 118 p.

Leal, M. and Losos, J. B. 2000. Bobos Chamaleolis barbatus and C. porcus. J. Herpetol. 34:318-322.

Márquez, C. 1994. Historia natural de A. aquaticus Taylor 1956 (Sauria:Polychridae) en la quebrada La Palma, Puriscal San José, Costa Rica. Tesis de Maestria UCR, C.R. $108 \mathrm{pp}$.

Márquez, C., Mora, J. M., Bolaños, F. y Rea, S. 2005. Aspectos de la biología poblacional en el campo de Anolis aquaticus, (Sauria: Polychridae) en Costa Rica. Ecol. Apl. Vol. 4 No 1 y 2, pp 59-69.
Taylor, E. H. 1956. A review of the lizards of Costa Rica Univ. Kansas Sci. Bull. 38: I322.

Tinkle, D. W. 1967. The life and demography of the side- blotched lizard, Uta stansburiana. Misc. Publ. Mus. Zool. Univ. Mich. 132:1-182.

Trivers, R. L. 1976. Sexual selection and resource accruing abilities in Anolis garmani. Evol. 30:253-269.

Valerio, C. E. 1991. La diversidad Biológica de Costa Rica. Editorial Heliconia. Fundación Neotrópica, San José, Costa Rica. 153 p.

Zar, J. H. 1984. Biostatistical analysis. Second Ed. Prentice -Hall, Englewood Cliffs, N.J. $718 \mathrm{p}$ 